\title{
Variability of Mechanical Properties of Collagen Membranes used in Dentistry
}

\author{
LOREDANA SANTO ${ }^{1 *}$, FABRIZIO QUADRINI ${ }^{1}$, DENISE BELLISARIO ${ }^{1}$, ANTONELLA POLIMENI ${ }^{2}$, ANNA SANTARSIERO ${ }^{3}$ \\ ${ }^{1}$ Dipartimento di Ingegneria Industriale, Università di Roma Tor Vergata, Rome, Italy \\ 2 Dipartimento di Scienze Odontostomatologiche e Maxillo Facciali, Sapienza Università di Roma, Rome, Italy \\ ${ }^{3}$ Dipartimento Ambiente e Salute, Istituto Superiore di Sanità, Rome, Italy
}

The aim of this study is proposing a combination of measurements to assess the functional variability of collagen membranes used in Guided Bone Regeneration (GBR) and Guided Tissue Regeneration (GTR) techniques. As far as clinical applications are concerned, a proper qualification is critical when deciding, among commercially available collagen membranes, upon the most appropriate one for each specific clinical case. Two commercially available collagen membranes, namely Collprotect ${ }^{\circledR}$ and Jason ${ }^{\circledR}$, were considered for the experimentation. After thickness and density measurements, the quasi-static behavior was studied for both membranes by means of conventional mechanical tests, i.e. tear and tensile tests, whereas their time-dependent behavior was evaluated by means of stress relaxation tests and dynamic mechanical analysis. Collagen membranes showed an elevated among samples variability. The variability within the same kind of membrane is of the same order of magnitude of the between membrane kinds variability. All the membranes showed strong time dependence both in stress relaxation and in dynamic mechanical tests. This fact should be taken under consideration for the membrane final application.

Keywords: collagen, membrane barrier; mechanical properties, thickness, guided tissue regeneration, guided bone regeneration

In this paper, carrying on a previous research developed by the authors [1], the variability of mechanical properties of collagen membranes used in GBR/GTR techniques is investigated. Collagen membranes used in dentistry typically show an elevated variability among samples $[1,2]$, therefore doubts arise about the possibility of a rational choice of a collagen membrane only on the basis of their mechanical performances. The relationship between mechanical properties and thickness is crucial when handling and placing the membrane on the defect site, and definitely so when having to choose, among the commercially available collagen membranes, the most appropriate one for each specific case.

The assessment of size and mechanical properties of natural collagen membranes has a big relevance for the massive use of GBR/GTR techniques. The use of membranes in GTR/GBR techniques has originated in the need of isolating the periodontal defect with a mat-like material that acts as a barrier [3, 4]. From a clinical point of view, this is accomplished by placing a barrier over the defect, between the epithelium and the tooth, therefore giving the cells of the periodontal ligament time and space for regeneration. In GBR procedures, membranes are normally applied in combination with a bone/graft material. The membrane is placed over a bony defect, which is filled with a bone-graft material that prevents the collapse of the membrane and works as an osteoconductive scaffold for the in-growth of bone cells (or bone precursor cells). The barrier membrane prevents the ingrowth of soft tissue into the defect area and the encapsulation of the bone graft material, thus enabling the bone regeneration.

GBR/GTR membranes are broadly divided into two groups, non-resorbable and resorbable, according to their degradation rates. Both kinds of collagen membranes have been used for many years in Guided Tissue Regeneration
(GTR) and Guided Bone Regeneration (GBR) techniques because of their biocompatibility [5-15]. The firstgeneration barrier membranes were made of nonresorbable materials like expanded polytetrafluorethylene (e-PTFE) and cellulose acetate or titanium. These membranes gave good results, but they had the disadvantage of the need of a secondary surgery for their removal with the risk of grafting site morbidity. Therefore, further developments proceeded in the direction of resorbable membranes, which are synthetic polymers such as poly-glycolides and poly-lactides (in the case of acidic degradation). Natural polymer collagens have been used for this aim as well.

GBR/GTR collagen membranes must exhibit several characteristics: biocompatibility to allow integration with the host tissues without eliciting inflammatory responses; proper degradation time matching the new tissue formation time; adequate mechanical and physical properties to allow placement in vivo, avoiding membrane collapse and performing barrier function [15]. Tailoring the lifetime of a GBR/GTR-membrane is fundamental to fulfil the goal of optimal tissue/bone regeneration, and various types of collagen membranes have been placed on the market [16]. In order to choose the most appropriate product for each specific case, a detailed knowledge of material properties and structures is needed to predict whether membranes will be able to outstand [17] expected loads during tissue/bone regeneration, till they biodegrade and are absorbed (minimum 4 weeks) $[18,19]$, not to mention the loads deriving from each step of their application (i.e. during handling and placing on the defect site).

Before application, membranes have to be customized to the defect. Thus they must be easy to handle: be fairly malleable to conform to the defect shape, and yet preserve their structure. They should be easy to cut and shape, with 
no sharp edges that may perforate tissues [20], and be flexible enough to allow close adaptation to a variety of defect morphologies [21]. Loads and scratches during clinical handling, before and during the membrane resorption, could affect membrane stability with the risk of losing the expected barrier properties, hence the importance of furnishing information about the mechanical behavior in relation to the thickness of a collagen membrane.

Membrane thickness may have implications during and after membrane placement on the defect site. The placement of a thin membrane on the defect site may lead to complications. For example, a thick gingival flap may exert pressure over the membrane and affect its stability. On the other side, one of the most frequent postoperative complications of GTR is membrane exposure that mostly occurs when the membrane is covered by a thin flap [22]. Therefore, adequate ûap and membrane thickness is crucial for the success of GBR/GTR procedure [23-27]. Membrane thickness higher than $0.8 \mathrm{~mm}$ in the defect area seems to have a great inûuence in improving the percent root coverage $(26.7 \%$ versus $95.9 \%$ of root coverage in thin and thick tissues, respectively) [23-24]. This result seems to be consolidated in the scientific background [25-27]. Moreover, thickness of gingival tissue covering a membrane is a factor to consider if posttreatment recession is to be minimized or avoided [2830].

From a clinical stand-point, these membranes are generally easy to handle and to place on the defect, but they have a limited capacity to maintain space for regeneration and to support gingival tissues. In this regard, the negative effect of an early membrane exposure on the regenerative outcome in guided tissue regeneration (GTR) and guided bone regeneration (GBR) procedures is somewhat controversial [31]. Membrane exposure during healing seems to have a major negative effect on GBR around dental implants but only a minimal effect on GTR around natural teeth [32]. Membrane exposure may also have a negative effect on healing, such as differences in revascularization. Recent studies have shown that a successful bone/tissue regeneration procedure using membranes depends on several factors including defect size, membrane biodegradation, position of the defect and membrane thickness [33].

Data on parameters involved in the performance-based design (e.g., material properties such as the elastic modulus, tensile stress etc., or dimensional parameters such as thickness) with their fixed range of variability, are crucial as they allow detecting acceptable levels of membrane damage during clinical handling and application.

Motivation for the current study is to be found in the huge difference in mechanical properties among the investigated [1,2] membranes. Particular attention is paid to inter- and intra-sample thickness variability. In fact, thickness can affect the actual performance of a given sample of a membrane, but, in itself, cannot inform about expected membrane performances. In this view, we have investigated the correlation between thickness and response under mechanical testing for two commercially available GTR/GBR collagen membranes, namely Collprotect ${ }^{\circledR}$ and J ason $\circledast$.

\section{Experimental part}

Materials and Methods

In this study, two types of collagen membranes, namely Collprotect ${ }^{\circledR}$ and Jason ${ }^{\circledR}$, by Botiss Biomaterials (Italy) were considered for the experimentation. As reported in the technical datasheets, Collprotect $₫$ is a natural collagen membrane originating from porcine dermis with a degradation time of approximately 8-12 weeks and a slight stiffness. Collprotect $\AA$, as reported by the manufacturer, is intended for middle-term barrier function in GBR/GTR procedures. Its structure is a dense network of collagen bundles with pores for better vascularization. J ason $₫$ is a native collagen membrane originating from porcine pericardium, designed and produced for GBR/GTR procedures. Datasheets report that it is characterized by a long-lasting barrier function for approximately $12-24$ weeks and a high adaptability during placement. Its structure consists of differently oriented collagen fibres also to provide multi-directional tear resistance.

Six samples were analyzed for each type of membrane. The nominal area of each sample was $15 \times 20 \mathrm{~mm}^{2}$, size of the product as supplied. After thickness and weight measurements, all the membrane samples were used for mechanical testing. Measurements were carried out in dry conditions to evaluate material properties in supplying state at which their mechanical response is expected to be maximum. Thickness measurements were performed by a digital caliper (Rupec 239, resolution $0.001 \mathrm{~mm}$, reference standard DIN 863/1). The caliper allows for reliable thickness measurements because minimum pressure applied over a large area results in negligible sample deformation. The nominal surface density was calculated as the ratio between the initial weight and the nominal surface area of the dry membranes.

From a sample of each membrane type, two specimens were extracted for dynamic-mechanical analysis: one along the main direction and the other in the orthogonal direction. Specimens had a nominal width of $2 \mathrm{~mm}$, and were tested in tensile configuration with a gage-length of $10 \mathrm{~mm}$. Dynamic-mechanical analysis was performed using a Netzsch 242C dynamic mechanical analyzer (DMA); and DMA tests were performed at room temperature under isothermal condition at a frequency of $10 \mathrm{~Hz}$, and for $10 \mathrm{~min}$. With DMA tests it is possible to recognize the elastic part of the material deformation (related to the storage modulus) and the plastic part (related to the loss factor). In the case of perfectly elastic materials, the loss factor is zero and all the deformation is elastic. Polymeric and natural organic materials are typically visco-elastic with a viscous behavior also at low strains and strain-rates; the more viscous the material, the higher the loss factor. Making tests along two orthogonal directions is a way for measuring the intrinsic anisotropy of the samples.

The quasi-static behavior of the membranes has been studied by means of conventional mechanical tests: tear and tensile tests. Apart from DMA, the time-dependent behavior of membranes was also evaluated by means of stress relaxation tests. From each of the other five remaining membrane samples, three specimens were extracted, one for each test: tear, tensile, and stress relaxation. All the mechanical tests were performed by using a universal testing machine (MTS Insight 5) with a load cell of $100 \mathrm{~N}$ and a crosshead speed of $1 \mathrm{~mm} / \mathrm{min}$.

Tear testing provides information on the energy or force required to propagate a tear through the material. In our case, we measured the tear-propagation resistance of the collagen membranes. Specimens were cut with a nominal area $7.5 \times 20 \mathrm{~mm}^{2}$, and the tear was initiated with a $7 \mathrm{~mm}$ long central cut according to figure 1a. Tear propagation was monitored as a function of the vertical displacement, at the constant rate of $1 \mathrm{~mm} / \mathrm{min}$, up to a maximum displacement of $10 \mathrm{~mm}$. 
Tensile tests and stress relaxation tests were performed on membrane specimens with an area of $3.5 \times 20 \mathrm{~mm}^{2}$. Tensile testing allows studying the mechanical behavior of materials subjected to a controlled tension until failure. During testing, a longitudinal strain was applied at constant rate along the main size of the rectangular or dog-bone shaped specimen, and the resulting force (or stress) was measured. Due to the small size of the membrane samples (about $15 \times 20 \mathrm{~mm}^{2}$ ), the rectangular shape was chosen with a size of $3.5 \times 20 \mathrm{~mm}^{2}$. Tests were performed with a gage length (i.e. distance between clamps) of $12 \mathrm{~mm}$ at a rate of $1 \mathrm{~mm} / \mathrm{min}$ up to a maximum displacement of 10 $\mathrm{mm}$ until specimen failure. For membranes, the properties that are directly measured via tensile test are elastic modulus, ultimate tensile strength and maximum elongation (or elongation to break). The stress relaxation test describes how materials release stresses under constant strain over time. In this study, the imposed strain value was fixed during a loading stage (i.e. it was equal to the strain to achieve a maximum stress of $2 \mathrm{MPa}$ ). The stress relaxation behavior was evaluated within a time span of $5 \mathrm{~min}$.

\section{Results and discussions}

All the data from experimental tests are summarized in table 1 in terms of mean and standard deviation. There was a large variability in thickness among the different samples of each membrane; in fact dispersion is $12 \%$ of the average value for Collprotect, and up to $39 \%$ for J ason. Making a comparison between membranes, the average thickness of the former is $0.278 \mathrm{~mm}$, higher than the average thickness of the latter, about $0.197 \mathrm{~mm}$. The nominal surface density is also reported in the table. The average surface density is $0.00886 \mathrm{~g} / \mathrm{cm}^{2}$ for Collprotect and $0.00362 \mathrm{~g} / \mathrm{cm}^{2}$ for J ason. Variability in density is comparable or lower than variability in thickness, but it is not negligible, especially for Collprotect.

Figures $1 b$ and $1 c$ report the tear test curves for Collprotect and Jason, respectively, and show also the filaments produced during tear test. All the curves show strong irregularity even if a general increasing trend is always recognizable up to a plateau. From each testa tear force was extracted, by averaging the acquired load in the 2-6 $\mathrm{mm}$ displacementrange, and plotted as shown in figure 2. Tear force was similar for all J ason specimens, whereas a high scattering was observed for Collprotect. Mean values of tear load for the two different membranes are reported in table 1.

Figure 3 shows the cell configuration for the tensile mode, and the results of DMA tests in isothermal condition. Storage modulus and loss factor data are reported as a function of the direction, being the two specimens extracted along two orthogonal directions. Direction is very important for the prediction of behavior during use, as anisotropy often affects the response of the materials under complex loading conditions. In our case, the effect of specimen orientation turned out to be negligible. In view of this planar isotropy, only the main direction was chosen for the extraction of the specimens for the mechanical tests (tear, tensile, and stress relaxation).

\begin{tabular}{|l|c|c|c|c|}
\hline & \multicolumn{2}{|c|}{ Collprotect membrane } & \multicolumn{2}{c|}{ Jason membrane } \\
\cline { 2 - 5 } & Average & Standard deviation & Average & Standard deviation \\
\hline Thickness [mm] & 0.278 & 0.034 & 0.197 & 0.077 \\
\hline Density [g/cm $\left.{ }^{3}\right]$ & 0.0089 & 0.0013 & 0.0036 & 0.0004 \\
\hline Tear load [N] & 1.15 & 0.50 & 0.74 & 0.08 \\
\hline Elastic modulus [MPa] & 158.46 & 35.82 & 178.86 & 133.99 \\
\hline $\begin{array}{l}\text { Maximum tensile stress } \\
{[\mathrm{MPa}]}\end{array}$ & 13.14 & 1.94 & 13.02 & 7.97 \\
\hline Maximum tensile strain $[\%]$ & 15.88 & 2.66 & 15.29 & 3.84 \\
\hline Stress relaxation [\%] & 17.00 & 2.65 & 22.00 & 4.04 \\
\hline
\end{tabular}

Table 1

DATA EXTRACTED FROM EXPERIMENTAL TESTS

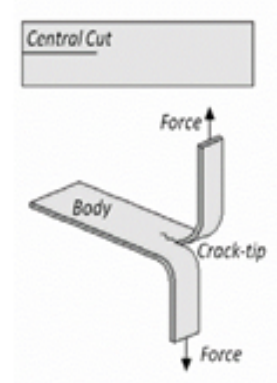

a)

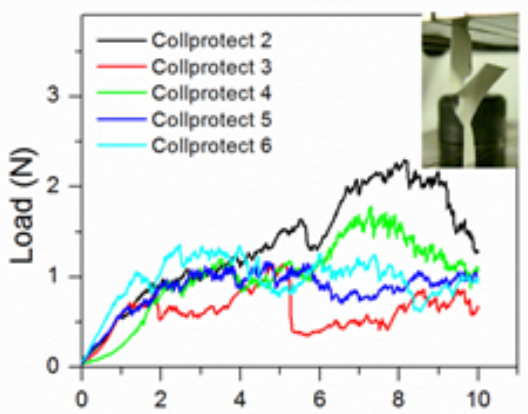

b)

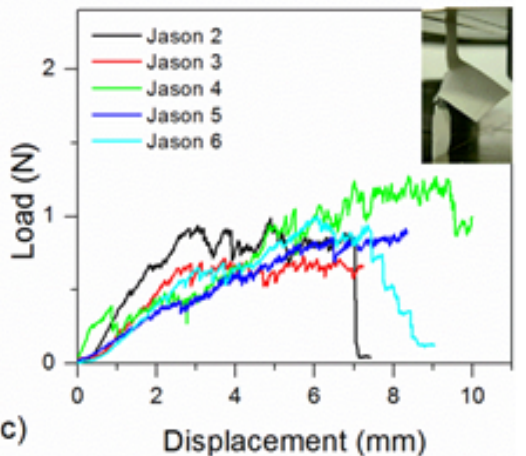

Fig. 1. Tear test configuration (a), Tear test curves of Collprotect

(b) and Jason (c) membrane samples.

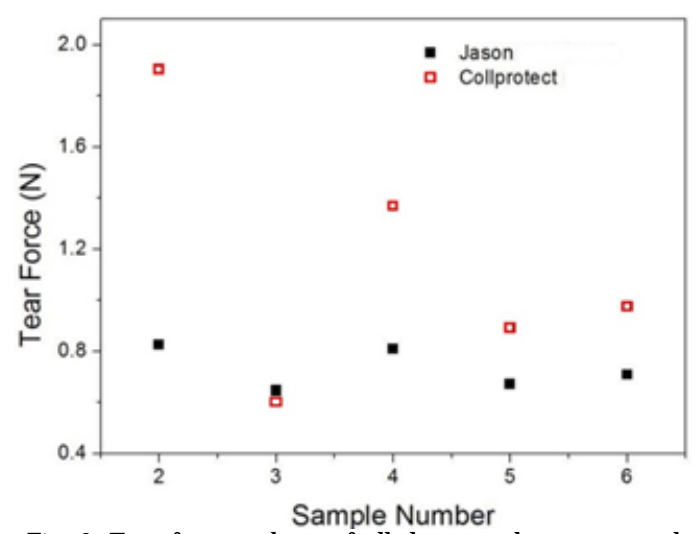

Figures $4 a$ and $4 b$ show tensile curves for Collprotect and Jason, respectively, and figure 5 reports the extracted elastic modulus for all specimens of both membranes. The average tensile modulus for Jason and Collprotect membranes is 179 and $158 \mathrm{MPa}$, respectively. Therefore, Jason membrane exhibited, on average, higher stiffness than Collprotectboth in dynamic (fig. 3) and in static tensile tests. However, the difference with Collprotect is quite lower in quasi-static tensile tests than in the case of the storage modulus by DMA.

Figure 6 reports the extracted maximum stress and strain for all the specimens of the membranes; and data scattering is very high for the extracted maximum stress

Fig. 2. Tear force values of all the membranes samples. 

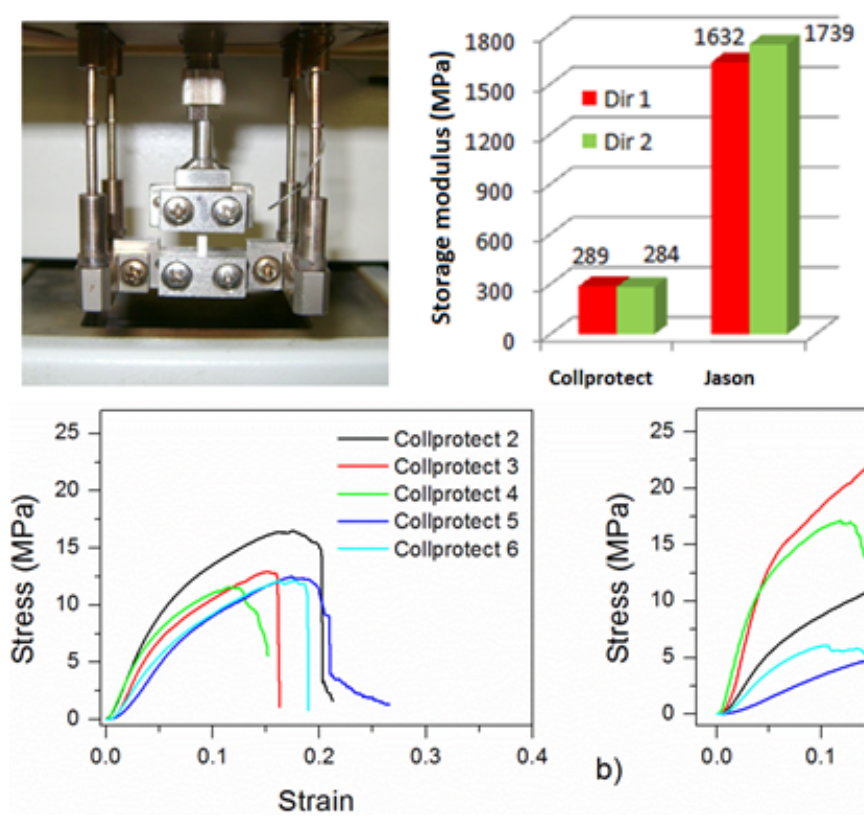

Collprotect Jason
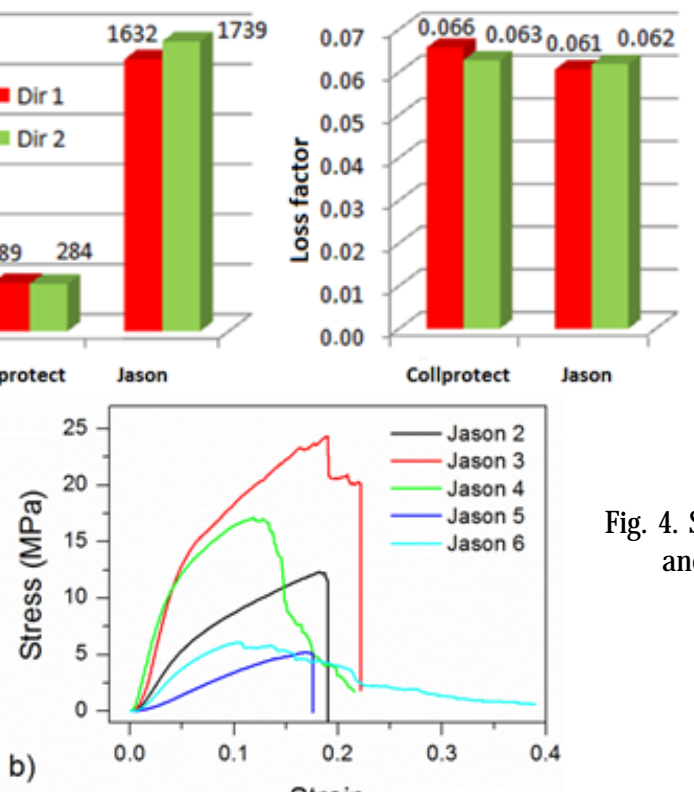

Fig. 3. Results of DMA tests in tensile mode and isothermal condition
Fig. 4. Stress-strain curves of Collprotect (a) and Jason (b) membrane samples.

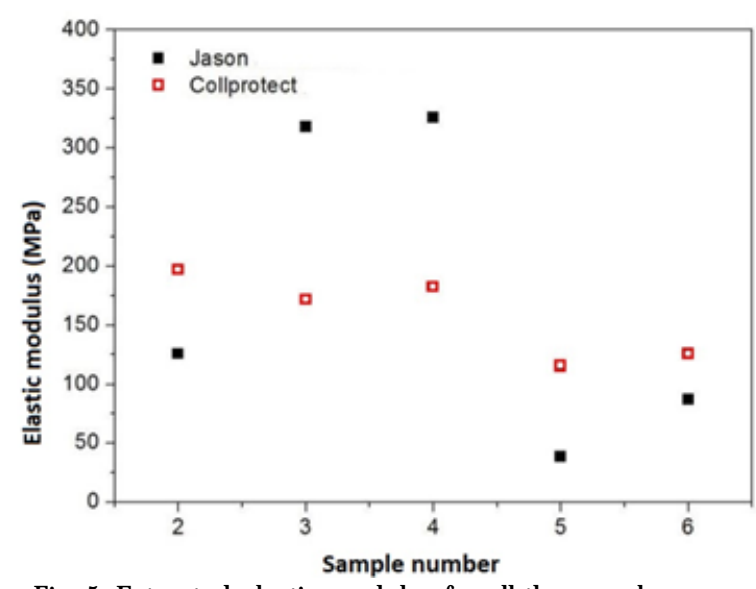

Fig. 5. Extracted elastic modulus for all the membrane samples.

and strain. Extracted elastic modulus and ultimate properties (maximum stress and strain) are also reported in table 1 in terms of average value and standard deviation.

Figures $7 \mathrm{a}$ and $7 \mathrm{~b}$ report the stress relaxation curves for Collprotect and Jason, respectively. Test curves confirm the variability in the material response for all the specimens. For comparison purpose, the stress reduction after $5 \mathrm{~min}$ was taken into consideration. On average (table 1) stress relaxation was higher in the stiffer J ason membrane, but again some specimens (\#3 and \#4) behaved in the range of the Collprotect membrane. This fact is highlighted in figure $7 c$, which shows the stress relaxation after $5 \mathrm{~min}$ for all the specimens of both membranes.

By measuring the thickness of membranes one can easily evaluate the intrinsic non-homogeneity of these products. Unluckily this evaluation is difficult to put in relation with the final performance of the products. A deviation was observed for each sample as the thickness was measured for all the specimens extracted from the same sample. Within a single sample, the thickness can
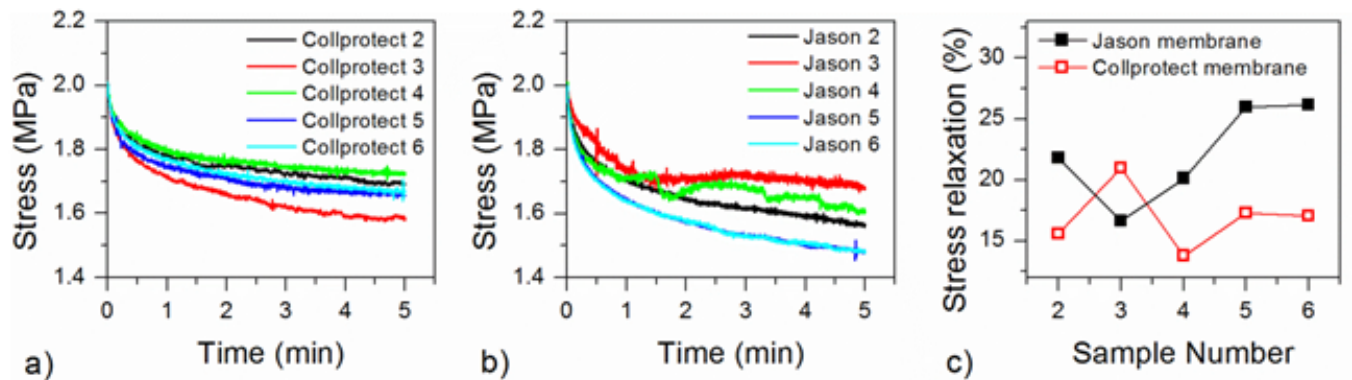

Fig. 7. Stress relaxation curves of Collprotect (a) and Jason (b) membrane samples, and stress relaxation rate $(\mathrm{c})$ for membrane samples 
$\left.\mathrm{cm}^{2}\right)$. Therefore, the thicker membrane showed the higher surface density, as expected because of the higher amount of material.

In order to understand whether differences in thickness and density could be related to differences in structure and performances, dynamic-mechanical, mechanical, and stress relaxation tests were performed. As shown in figure 3 , the higher rigidity of the Jason membrane (produced from pericardium) is evident, in comparison with Collprotect (produced from dermis). The storage modulus of Jason membrane is five times that of Collprotect membrane, and is also high in absolute terms, in comparison with typical unfilled organic compounds. Instead, the loss factor is comparable for both types of membranes, and is generally low.

The complex nature of collagen membranes is also clearly visible during tear tests as specimens tend to produce filaments during crack propagation (fig. $2 \mathrm{~b}$ and 2c). Many times, during testing, crack propagation stopped because of failure of a leg. For both membranes, the shape of the tear test curves is strongly irregular. Irregularities are typical for this kind of tests but they were exalted by the non-coherent structure of the membranes. Generally, after an initial increasing part, tear test curves reach a plateau and the average force in this plateau can be used as a measurement of the tear force.

Despite of high scattering in thickness, the tear force is similar for all the samples of ] ason, whereas high scattering is still visible for Collprotect (fig. 2 and table 1). It is interesting to observe that Jason was stiffer under tensile load in DMA, but its average tear force is lower than Collprotect. Dealing with conventional materials, typically stiffer materials also exhibit higher strengths but that is not a rule as elastic properties (e.g. by DMA) and ultimate properties (such as tear load or tensile strength) are conceptually not correlated. Data scattering for tear force of Collprotect specimens is quite high, however as shown by figure 2, a single specimen (\#3) was found to be worse than all the Jason specimens.

Data scattering has been observed also in tensile tests under quasi-static loading (fig. 4). A double-linear trend is generally visible for all the specimens and an elastic modulus was extracted from the first linear stage (table 1). Figure 5 reports the extracted elastic modulus for all specimens of both membranes. Even if the Jason membrane exhibited, on average, a higher stiffness also in static tensile tests, the difference with Collprotect is quite lower than in the case of the storage modulus by DMA. Figure 6 reports the extracted maximum stress and strain for all the specimens of the membranes. Data scattering is very high.

If only average data of tensile tests are considered, very similar results can be observed for both Collprotect and Jason, but the real issue is given by the standard deviation which is high for both materials and higher for the latter (except for the tear load). This large variability was expected also in stress relaxation tests, and it is in fact confirmed by figure 7 showing stress relaxation curves for Collprotect and Jason membranes, respectively. A low stress was used in the test ( $2 \mathrm{MPa}$ ) but it was sufficient to observe a high stress relaxation for all the specimens at low times. In order to quantify the stress relaxation, the load decrease after 5 min was extracted and normalized by using the initial stress. On average, stress relaxation was higher in the stiffer Jason membrane but also in this case, some specimens (\#3 and \#4) behaved in the range of the Collprotect membrane (fig. 7c). Deformation mechanisms could play an important role in the definition of the tear properties as well as the stress relaxation behavior. In any case, stress relaxation is never lower than $12 \%$ in all the tested samples; as a result, these collagenbased materials are strongly sensitive to the effect of time at fixed loads.

\section{Conclusions}

Mechanical properties of membranes are strongly affected by the architecture of the membrane, and data scattering could be related to the non-uniformity of the material structure as well as the sample thickness. In general, results of this study, in which a higher number of samples were tested, confirm findings of previous investigated collagen membranes [1,2]. Samples of the same membrane behaved in different ways under the same tests (i.e., tensile, tear and stress relaxation testing). This result highlights that the membrane sample thickness (or density in this case) plays an important role in their final performance, at least in terms of uniformity. Comparing membranes in terms of average values is only partially useful as single samples of the same membrane type could behave better or worse than single samples of the other membrane type. The among samples variability is sometimes greater than between membranes variance. This lack of uniformity (probably due to the difficulties in the standardization of collagen extraction) partially inhibits the rational evaluation of membrane products. From a practical clinical point of view, all technical data should be reported on the leaflet inside the membrane package with all the nominal values and related fixed range of data variability concerning the membrane in both dry and wet conditions, so that the clinician may take them into consideration when choosing a membrane.

Acknowledgments: We gratefully acknowledge Dr Emanuela Ortolani for her continued interest in our work.

\section{References}

1. ORTOLANI E., et. al., Ann. Ist. Sup., 51, nr. 3, 2015, p. 229;

2. COÏC M., PLACET V., JACQUET E., MEYER C., Rev. Stomatol. Chir. Maxillofac., 111, nr. 5-6, 2010, p. 286;

3. KARRING T., NYMAN S., GOTTLOW J., LAURELL L., J. Periodontol., 1, nr. 1, 2000, p. 26;

4. MAGNUSSON I., BATICH C., COLLINS B.R., J. Periodontol., 59, nr. 1, 1988, p. 1;

5. POLIMENI G., et. al., Clin. Implant Dent. Relat. Res., 10, 2008, p. 99; 6. SCULEAN A., NIKOLIDAKIS D., SCHWARZ F., J. Clin. Periodontol., 35, nr. s8, 2008, p. 106;

7. BEHRING J., JUNKER R., WALBOOMERS X.F., CHESSNUT B., J ANSEN

J.A., A systematic review. Odontology, 96, 2008, p. 1;

8. GEURS N.C., et. al., J. Periodontol., 79, 2008, p. 1133;

9. PIATTELLI A., SCARANO A., RUSSO P., MATARASSO S., Biomaterials, 17, 1996, p. 791;

10. NYMAN S., GOTTLOW J., KARRING T., LINDHE J., J. Clin. Periodontol., 9, 1982, p. 257;

11. NYMAN S., LINDHE J., KARRING T., RYLANDER H., J. Clin. Periodontol., 9, 1982, p. 290;

12.NYMAN S., GOTTLOW J ., LINDHE J ., KARRING T., WENNSTROM J ., J. Periodontal Res., 22, 1987, p. 252;

13. LINDE A. et. al., J. Periodontol., 64, 1993, p. 1116;

14.BOTTINO M.C., et. al., Dent. Mater., 28, 2012, p. 703;

15. NYMAN S., J. Clin. Periodontol, 18, nr. 6, 1991, 494;

16. WILLERSHAUSEN I. et. al., J. Appl. Oral Sci., 22, nr. 1, 2014, p. 29; 17.SANTARSIERO A., Roma: Istituto Superiore di Sanità; 2013. (Rapporti ISTISAN 13/22).

18.BOZKURT A., et. al., Clin. Oral. Impl. Res., 2013, p. 1;

19. BOTTINO M. C., THOMAS V., JANOW SKI G.M., Acta Biomater., 7, 2011, p. 216; 
20. T.V. SCANTLEBURY, 1982-1992: A decade of technology development for guided tissue regeneration, J. Periodontol. 64 (1993) 1129-1137.

21. WANG H.L., MacNEIL R.L., Dent. Clin. North Am., 42, 1998, p. 505;

22. GOTTLOW J., NYMAN S., Curr. Opin. Periodontol., 3, 1996, p. 140;

23. WANG H., KIMBLE K., EBER R., Int. J. Periodontics Restorative Dent., 22, 2002, p. 119;

24.KIMBLE K., EBER R., SOEHREN S., SHYR Y., WANG H., J. Periodontol., 75, 2004, p. 210;

25. JEPSEN S., HEINZ B., KERMANIE M.A., JEPSEN K., J. Periodontol., 71, 2000, p. 1433;

26.HARRIS R.J ., J. Periodontol., 68, 1997, p. 779;

27.BALDI C., et. al., J. Periodontol., 70, 1999, p. 1077;
28.ANDEREGG C.R., METZLER D.G., NICOLL B.K., J. Periodontol., 66, nr. 5, 1995, p. 397;

29.MÜLLER H.P., KONONEN E., J. Periodontal. Res., 40, nr. 3, 2005, p. 239;

30.WENNSTRÖM J.L., ZUCCHELLI G., J . Clin. Periodontol., 23, nr. 8, 1996, p. 770;

31.MACHTEI E.E., J. Periodontol., 72, nr. 4, 2001, p. 512;

32.HITTI R.A., Kerns D.G., Open Pathol. J., 5, 2011, p. 33;

33.SOHEILIFAR S., BIDGOLI M., TORKZABAN P., Avicenna J. Dent. Res., 6, nr. 2, 2014, e21343.

$\overline{\text { Manuscript received: } 15.09 .2018}$ 